\title{
Comunicação em saúde por uma população adscrita de uma clínica da família do
}

\section{município do Rio de Janeiro}

\author{
Health communication by an ascribed population of a family clinic in the city of Rio de Janeiro \\ Comunicación de salud de una población inscrita de una clínica familiar en la ciudad de Río de
}

Janeiro

Recebido: 25/03/2021 | Revisado: 03/04/2021 | Aceito: 08/04/2021 | Publicado: 18/04/2021

Sara Soares Ferreira da Silva

ORCID: https://orcid.org/0000-0001-8754-4438 Universidade Federal do Estado do Rio de Janeiro, Brasil

E-mail: sarasferreira@edu.unirio.br

Danielle Galdino de Paula

ORCID: https://orcid.org/0000-0002-0103-6828 Universidade Federal do Estado do Rio de Janeiro, Brasil E-mail: danielle.paula@unirio.br

Marina Medeiros Ribeiro

ORCID: https://orcid.org/0000-0002-6732-4646 Universidade Federal do Estado do Rio de Janeiro, Brasil E-mail: ribeiromarina89@gmail.com

Yuri Zoel Brasil

ORCID: https://orcid.org/0000-0002-9130-9278 Pontifícia Universidade Católica do Rio de Janeiro, Brasil E-mail: yuri.zoel@gmail.com

\begin{abstract}
Resumo
O estudo objetiva apresentar subsídios para o desenvolvimento de estratégias eficazes de comunicação em saúde para uma população adscrita de uma unidade básica de saúde do município do Rio de Janeiro através da realização de um diagnóstico situacional. Trata-se de estudo transversal, descritivo e de abordagem quantitativa, com utilização de dados secundários oriundos de 200 relatórios produzidos na unidade de saúde. Foram adquiridas informações sobre perfil sociodemográfico e processo de comunicação em saúde da população adscrita. A mediana de idade dos participantes foi de 35 anos, sendo a maioria do sexo feminino. O nível de escolaridade predominante foi ensino fundamental incompleto e renda de um salário mínimo. A maioria da população possuía smartphones, televisão e acesso à internet. Foi verificado que os usuários possuem o Google® e Profissionais de saúde como fontes de informação em saúde recorridas, sendo o Google $₫$ mais expressivo entre pessoas do sexo masculino em detrimento do sexo feminino, que utiliza mais o Instagram ${ }^{\circledR}$. No que se refere às estratégias de comunicação em saúde, a idade se mostrou como fator estatisticamente relevante quanto à aceitação de panfletos informativos sobre saúde $(\mathrm{p}=0,005)$. Quanto às fake news sobre saúde, foi verificado que participantes de idade entre 18 a 25 anos valorizam mais a checagem de informações, contudo $85 \%$ dos usuários afirmaram a importância de tal procedimento. Portanto, os achados contribuem para compreensão do processo de comunicação em saúde e planejamento de ações educacionais por profissionais assistenciais da Atenção Primária à Saúde, evidenciando seu importante papel neste contexto.
\end{abstract}

Palavras-chave: Comunicação em saúde; Educação em saúde; Atenção primária à saúde; Enfermagem.

\begin{abstract}
The study aims to present subsidies for the development of effective health communication strategies for an enrolled population of a basic health unit in the city of Rio de Janeiro through the realization of a situational diagnosis. This is a cross-sectional, descriptive study with a quantitative approach, using secondary data from 200 reports produced at the health unit. Information was acquired on the sociodemographic profile and health communication process of the population enrolled. The median age of the participants was 35 years old, the majority being female. The predominant level of education was incomplete primary education and income of a minimum wage. Most of the population had smartphones, television and internet access. It was found that users have Google ${ }^{\circledR}$ and Health Professionals as sources of health information used, with Google ${ }^{\circledR}$ being more expressive among men over women, who use Instagram ${ }^{\circledR}$ more. With regard to health communication strategies, age was shown to be a statistically relevant factor regarding the acceptance of health information pamphlets $(\mathrm{p}=0,005)$. As for fake health news, it was found that participants aged 18 to 25 years old value information checking more, however $85 \%$ of users affirmed the importance of such a procedure. Therefore, the findings contribute to the understanding of the health communication process and the planning of educational actions by primary health care professionals, highlighting its important role in this context.
\end{abstract}


Keywords: Health communication; Health education; Primary health care; Nursing.

\section{Resumen}

El estudio tiene como objetivo presentar subsidios para el desarrollo de estrategias efectivas de comunicación en salud para una población inscrita en una unidad básica de salud de la ciudad de Río de Janeiro mediante la realización de un diagnóstico situacional. Se trata de un estudio descriptivo transversal con enfoque cuantitativo, utilizando datos secundarios de 200 informes elaborados en la unidad de salud. Se obtuvo información sobre el perfil sociodemográfico y el proceso de comunicación en salud de la población inscrita. La edad media de los participantes fue de 35 años, la mayoría mujeres. El nivel de educación predominante fue la educación primaria incompleta y el ingreso de un salario mínimo. La mayoría de la población tenía teléfonos inteligentes, televisión y acceso a Internet. Se encontró que los usuarios tienen a Google ${ }^{\circledR}$ y a los Profesionales de la Salud como fuentes de información de salud utilizadas, siendo Google ${ }^{\circledR}$ más expresivo entre los hombres que entre las mujeres, quienes usan más Instagram ${ }^{\circledR}$. En cuanto a las estrategias de comunicación en salud, la edad se mostró como un factor estadísticamente relevante en la aceptación de folletos de información en salud $(\mathrm{p}=0,005)$. En cuanto a las noticias de salud falsas, se encontró que los participantes de entre 18 y 25 años valoran más la verificación de información, sin embargo, el $85 \%$ de los usuarios afirmó la importancia de tal procedimiento. Por tanto, los hallazgos contribuyen a la comprensión del proceso de comunicación en salud y la planificación de acciones educativas por parte de los profesionales de la atención primaria de salud, destacando su importante papel en este contexto.

Palabras clave: Comunicación en Salud; Educación en Salud; Atención primaria de salud; Enfermería.

\section{Introdução}

A Atenção Primária em Saúde (APS) é considerada a forma mais adequada de acesso da população ao sistema de saúde, sendo responsável por contribuir para promoção de equidade entre indivíduos em uma sociedade. Dentre suas diversas atuações está inserida a educação em saúde definida como a transmissão de conhecimento com abordagem além do aspecto curativo, buscando englobar a prevenção e promoção da saúde, devendo ser adaptada aos diferentes contextos da população adscrita (Coscrato \& Bueno, 2013; Brasil, 2017; Starfield, Shi, \& Macinko, 2005; Tasca et al., 2020).

Devido a características inerentes, como integralidade, população adscrita, cuidado centrado na pessoa e longitudinalidade do cuidado, a APS se revela com amplo potencial para realização de ações de educação em saúde com imponente impacto. Contudo, para o desenvolvimento do processo ensino-aprendizagem, são necessárias ferramentas que permitam elaboração de estratégias e sua implementação de forma a gerar resultados satisfatórios, sendo uma destas ferramentas o conhecimento sobre a população ou grupo ao qual se pretende alcançar.

Entretanto, atualmente, novos desafios são impostos para educação em saúde na APS devido a mudança na forma de comunicação que vem ocorrendo de forma acelerada nos últimos anos. O Relatório da Global Digital demonstra que no ano de 2018 havia em torno de 4 bilhões de usuários de Internet no mundo, ou seja, mais da metade da população mundial se encontrava online. No Brasil, em 2018, 139,1 milhões de habitantes acessaram a Internet e $85 \%$ a utilizaram diariamente. Dentre os principais sites acessados destacam-se o Google, Facebook e YouTube e dentre as atividades mais realizadas na Internet estão "pesquisas" e "assistir vídeos" (We Are Social, 2018).

Tais dados ilustram como o modo de acesso à informação sofreu alteração nos últimos anos, o que antes era acessado através da televisão, rádios ou jornais impressos, hoje é majoritariamente acessado através de plataformas que permitem a livre escolha do tema e do transmissor do conteúdo (Miranda \& Rocha, 2018; We Are Social, 2018).

Estas alterações impactam diretamente na forma a qual a saúde deve ser comunicada atualmente para se mostrar eficaz. Na $15^{\text {a }}$ Conferência em Saúde, ocorrida no ano de 2015, a Informação, Educação e Política de Comunicação do Sistema Único de Saúde (SUS) foi objeto de discussão entre os participantes na qual destacou-se a importância da promoção e disseminação do conhecimento científico e tecnológico mediante entendimento da realidade da população atendida com inclusão de ações inovadoras saúde, contribuindo para a sustentabilidade do SUS. Outro aspecto foi a valorização do SUS como política de Estado, por meio de estratégias de comunicação tendo como eixo norteador a Educação Popular em Saúde (Ministério da Saúde, 2015; Brasil, 2013; Souza \& Cabral, 2014). 
É importante considerar que um desafio a ser superado é o crescimento das chamadas Fake News que podem ser entendidas como "notícias de baixa qualidade com informações intencionalmente falsas". Estas, em tempos de avanços tecnológicos, se disseminam rapidamente entre a população viabilizando ações contrárias às orientações das autoridades técnicas no campo da saúde (Silva et al., 2020; Neto et al., 2020; Shu et al., 2017).

Neste sentido, a APS possui potencialidade e o desafio de intervir no sentido de informar os usuários quanto a melhor forma de selecionar fontes de informação em saúde assim como desmistificar informações de conteúdo duvidoso disseminadas em seu território de abrangência, atuando como norteadora de informações em saúde de sua população adscrita, sendo uma importante forma de realizar educação em saúde nos tempos atuais.

Diante de diversos desafios a serem superados para realização de ações em educação em saúde eficientes pela APS, é necessário compreender como ocorre a dinâmica de acesso à informação e a forma pela qual a população interage com tais dados. Ademais, fatores concernentes aos contextos social, econômico e cultural precisam ser considerados, assim como a maneira ao qual se estabelece a comunicação entre profissional e usuário precisa sofrer processo de adaptação, de forma que sem tal flexibilização o cuidado que se pretende alcançar pode ser colocado em risco.

Desta forma, é de extrema importância o conhecimento sobre o processo de comunicação em saúde da população a qual se possui responsabilidade sanitária devendo este ser construído continuamente para que medidas eficientes de educação em saúde sejam propostas.

Portanto, o estudo tem como objetivo principal apresentar subsídios para o desenvolvimento de estratégias eficazes de comunicação em saúde para uma população adscrita de uma clínica da família do município do Rio de Janeiro, sendo seus objetivos específicos: Identificar os aspectos sociodemográficos da população e; Realizar um diagnóstico situacional sobre o processo de comunicação em saúde da referida população adscrita.

\section{Metodologia}

Trata-se de um estudo transversal, descritivo e de abordagem quantitativa (Medronho et al., 2008). O cenário do estudo foi uma unidade básica de saúde que atua sob os moldes de Estratégia de Saúde da Família, localizada na zona sul do município do Rio de Janeiro.

Foram utilizados dados secundários advindos de relatórios semanais gerados pela equipe de Estratégia de Saúde da Família da unidade de saúde do município do Rio de Janeiro. Os relatórios tinham por objetivo realizar um levantamento das fontes de informação utilizadas pelos usuários que procuravam atendimento na Unidade e tinham por objetivo nortear processos de comunicação entre usuários e profissionais da Unidade de Saúde. Os dados advindos dos relatórios foram coletados pela acadêmica de enfermagem, durante estágio extracurricular, sob supervisão da Enfermeira responsável técnica da Unidade. Estes dados foram analisados no período de 60 dias após aprovação dos Comitês de Ética em Pesquisa das instituições proponente e coparticipante.

Foi elaborado um instrumento de sistematização dos dados secundários, sendo este composto por duas etapas. Em uma primeira etapa foram captadas informações relacionadas ao perfil socioeconômico do participante (idade, gênero, renda média mensal individual, condições de saneamento básico, escolaridade, itens de comunicação, acesso à internet e sua qualidade) a fim de realizar correlações estatísticas de determinados grupos e analisar dados referente à população usuária do cenário de estudo. Na segunda etapa foram coletadas informações relacionadas ao perfil de acesso à comunicação consistindo em dados sobre as ferramentas de acesso à elucidação de temas de saúde (redes sociais, jornais, TV etc.), contando com diferentes estratégias empregadas na educação em saúde, fontes de informações em saúde, crenças sobre as informações recebidas por profissionais de saúde e realização meios de checagem de informações recebidas. Ressalta-se que os relatórios não continham informações de identificação do usuário. 
A referida unidade de saúde conta com quatro equipes de estratégia de saúde da família sendo cada uma denominada no presente estudo com códigos afim de manter seu anonimato sendo, portanto, identificadas como Equipe A, B, C e D.

Os dados secundários foram tabulados no programa Microsoft Excel® (versão 2016) e a análise realizada através dos softwares R commander versão 3.5.0® e Microsoft Excel®. Para variáveis quantitativas foi realizada análise exploratória e testes de hipótese não paramétricos (Qui-quadrado, Kruskal-Wallis e Exato de Fisher) devido a amostra não apresentar distribuição normal, sendo empregado nível de significância de 0,05 (Morettin \& Bussab, 2010).

Como critérios de inclusão foram considerados: registros compreendidos de julho a setembro de 2020; dados de pacientes com idade a partir de 18 anos e vinculados à unidade de saúde (cenário da pesquisa). Os critérios de exclusão foram: registros de dados incompletos e registros fora do período elencado no presente estudo. A partir de tais critérios a amostra analisada foi de 200 relatórios. Estes relatórios foram relativos ao período de estágio extracurricular.

Em atendimento a Resolução nº66/2012 do Conselho Nacional de Saúde o estudo foi submetido ao Comitê de Ética em Pesquisa (CEP/Brasil) da instituição proponente, Universidade Federal do Estado do Rio de Janeiro, e a instituição coparticipante, Secretaria de Saúde do Município do Rio de Janeiro (SMS/RJ) sendo aprovado pelo parecer nº 4.353 .106 e CAAE: 36078620.4 .3001 .5279 .

\section{Resultados}

Participaram da pesquisa 200 usuários da UBS, a mediana de idade foi de 35 anos e a média de $37,8(\sigma=14,6)$, no que se refere ao sexo biológico 153 (76,5\%) eram do sexo feminino e 47 (23,5\%) do sexo masculino. A distribuição de idade demonstrou que a maioria dos participantes eram do sexo feminino e estavam na faixa etária de 47 anos e do sexo masculino na faixa de 56 anos.

Ainda, foram coletados dados relacionados aos serviços utilizados pelos usuários na unidade básica de saúde, sua frequência de acesso à unidade e distribuição dos usuários quanto às equipes de saúde, visto que o cenário do estudo se trata de uma unidade sob moldes de Estratégia de Saúde da Família e, com vistas a subsidiar o planejamento de estratégias de educação em saúde para a população adscrita da UBS, foram coletados dados acerca do perfil de acesso à internet e a aparelhos de Tecnologia da Informação e Comunicação. Os referidos dados são ilustrados na Tabela 1.

Tabela 1. Perfil sociodemográfico, perfil de utilização de uma unidade de saúde e acesso às tecnologias da informação e comunicação da população adscrita de uma unidade básica de saúde, Rio de Janeiro, RJ, Brasil, 2020.

\begin{tabular}{lcc}
\hline \multicolumn{1}{c}{ Variáveis } & $\mathbf{n}$ & $\%$ \\
\hline Faixa etária & 70 & 35,0 \\
$18-29$ & 50 & 25,0 \\
$30-39$ & 61 & 30,5 \\
$40-59$ & 19 & 9,5 \\
60 anos ou mais & 200 & 100,0 \\
Total & & \\
Escolaridade & 61 & 30,5 \\
Ensino fundamental incompleto & 32 & 16,0 \\
Ensino fundamental completo & 35 & 17,5 \\
Ensino médio incompleto & 50 & 25,0 \\
Ensino médio completo & 16 & 8,0 \\
Ensino superior incompleto & & \\
\hline
\end{tabular}


Research, Society and Development, v. 10, n. 4, e45810414279, 2021

(CC BY 4.0) | ISSN 2525-3409 | DOI: http://dx.doi.org/10.33448/rsd-v10i4.14279

\begin{tabular}{|c|c|c|}
\hline Ensino superior completo & 6 & 3,0 \\
\hline \multicolumn{3}{|l|}{ Renda por salário-mínimo } \\
\hline Menos que 1 salário-mínimo & 73 & 36,5 \\
\hline 1 salário-mínimo & 88 & 44,0 \\
\hline 2 salários-mínimos & 32 & 16,0 \\
\hline 3 salários-mínimos & 5 & 2,5 \\
\hline 4 ou mais salários-mínimos & 2 & 1,0 \\
\hline \multicolumn{3}{|l|}{ Serviços básicos disponíveis na moradia } \\
\hline Água encanada & 194 & 97,0 \\
\hline Luz elétrica & 196 & 98,0 \\
\hline Tratamento de esgoto & 160 & 80,0 \\
\hline Gás encanado ou em botijão & 189 & 94,5 \\
\hline \multicolumn{3}{|l|}{ Equipes da unidade de saúde } \\
\hline A & 46 & 23,0 \\
\hline B & 58 & 29,0 \\
\hline $\mathrm{C}$ & 30 & 15,0 \\
\hline $\mathrm{D}$ & 45 & 22,5 \\
\hline Dado não disponibilizado & 21 & 10,5 \\
\hline \multicolumn{3}{|l|}{ Perfil de utilização da Clínica da Família } \\
\hline Acompanhamento de Hipertensão Arterial & 53 & 18,79 \\
\hline Demais motivos $^{1}$ & 49 & 17,38 \\
\hline Acompanhamento de Diabetes & 30 & 10,65 \\
\hline Consulta devido a sintomas agudos & 29 & 10,28 \\
\hline Pré-natal & 28 & 9,93 \\
\hline Puericultura & 27 & 9,57 \\
\hline Informação não disponibilizada & 21 & 7,45 \\
\hline Acompanhamento de Ansiedade/Depressão & 18 & 6,39 \\
\hline Vacinação & 14 & 4,96 \\
\hline Acompanhamento de saúde bucal & 5 & 1,77 \\
\hline Realização de exames & 5 & 1,77 \\
\hline Administração de medicamentos para contracepção & 3 & 1,06 \\
\hline \multicolumn{3}{|l|}{ Frequência de procura à Clínica da Família } \\
\hline Uma vez por semana & 22 & 11,0 \\
\hline Uma vez a cada 15 dias & 15 & 7,5 \\
\hline Uma vez por mês & 62 & 31,0 \\
\hline Uma vez a cada 3 meses & 18 & 9,0 \\
\hline Uma vez a cada 6 meses & 16 & 8,0 \\
\hline Uma vez ao ano & 22 & 11,0 \\
\hline Todos os dias & 6 & 3,0 \\
\hline Esporadicamente $^{2}$ & 21 & 10,5 \\
\hline Informação não disponibilizada & 18 & 9,0 \\
\hline \multicolumn{3}{|l|}{ Acesso a aparelhos eletrônicos } \\
\hline Smartphone (aparelho celular) & 186 & 36,97 \\
\hline Televisão & 183 & 36,38 \\
\hline Computador & 57 & 11,3 \\
\hline
\end{tabular}


Research, Society and Development, v. 10, n. 4, e45810414279, 2021

(CC BY 4.0) | ISSN 2525-3409 | DOI: http://dx.doi.org/10.33448/rsd-v10i4.14279

\begin{tabular}{|c|c|c|}
\hline Notebook & 48 & 9,5 \\
\hline Tablet & 27 & 5,3 \\
\hline Nenhum item citado & 2 & 0,55 \\
\hline \multicolumn{3}{|c|}{ Acesso à Internet no domicílio por conexão Wi-Fi } \\
\hline Possui & 156 & 78,0 \\
\hline Não possui & 44 & 22,0 \\
\hline \multicolumn{3}{|c|}{ Acesso à Internet no aparelho celular por dados móveis } \\
\hline Possui & 119 & 59,5 \\
\hline Não possui & 81 & 40,5 \\
\hline \multicolumn{3}{|c|}{ Qualidade da Internet domiciliar (conexão Wi-Fi) ${ }^{3}$} \\
\hline Excelente & 41 & 26.45 \\
\hline Muito bom & 52 & 33.55 \\
\hline Bom & 36 & 23.23 \\
\hline Médio & 17 & 10.97 \\
\hline Ruim & 2 & 1,29 \\
\hline Péssimo & 8 & 4,51 \\
\hline \multicolumn{3}{|c|}{ Qualidade da Internet no aparelho celular (dados móveis) } \\
\hline Excelente & 45 & 37,8 \\
\hline Muito bom & 28 & 23,5 \\
\hline Bom & 30 & 25,2 \\
\hline Médio & 12 & 10,1 \\
\hline Ruim & 1 & 0,88 \\
\hline Péssimo & 3 & 2,52 \\
\hline
\end{tabular}

Legenda: 1- Acompanhamento de familiar durante atendimento, doença renal, distúrbio de tireoide, agravo hepático, agravo neurológico, acompanhamento psiquiátrico/psicológico, dispensação de medicamentos, câncer, realização de curativo e tratamento de tuberculose.

2 - Usuário que utiliza a clínica somente para ações pontuais de saúde, ex: campanha de vacinação.

3 - Classificações realizadas conforme percepção dos usuários se tratando, portanto, de dados subjetivos.

Fonte: Dados do estudo.

Ademais, os participantes apontaram as informações em saúde que mais despertam o interesse, sendo os resultados demonstrados na Figura 1. 
Figura 1. Temas em saúde de maior interesse da população estudada. Rio de Janeiro, RJ, Brasil, 2020.

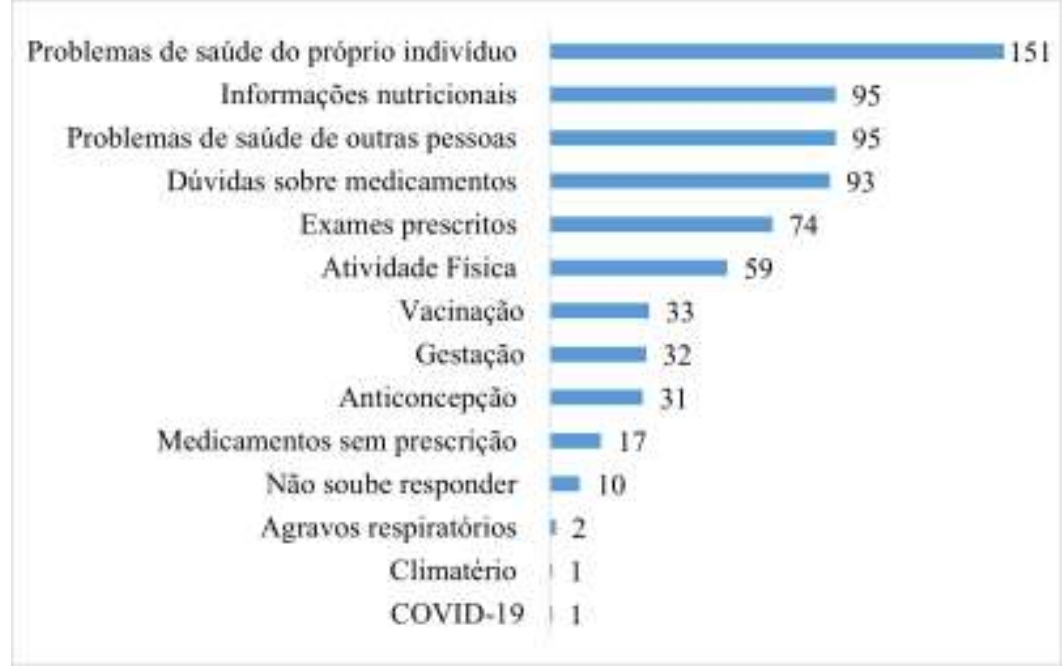

Fonte: Dados do estudo.

Outro aspecto abordado consistiu no maior entendimento sobre a relação estabelecida entre os usuários da unidade de saúde e as fontes de informações empregadas para aquisição de conhecimentos em saúde, tanto no que se refere aos momentos cotidianos como em momentos sintomáticos não críticos, como por exemplo apresentação de cefaleia branda, coriza, etc. A Figura 2 demonstra as fontes utilizadas em ambas as situações.

Figura 2. Fontes de informações em saúde utilizada em momentos sintomáticos e no cotidiano da população adscrita. Rio de Janeiro, RJ, Brasil, 2020.

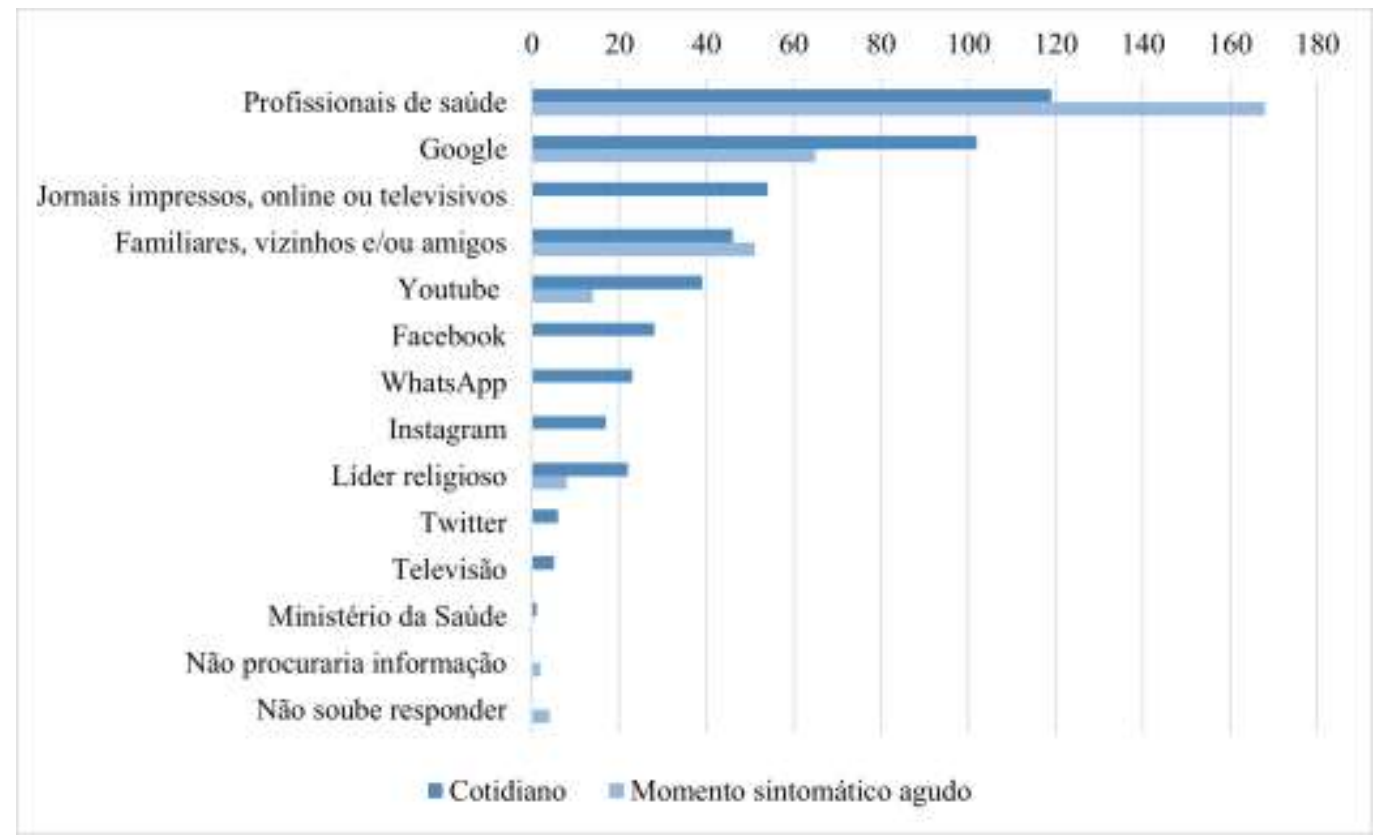

Fonte: Dados do estudo.

Quanto ao sexo biológico dos participantes, as fontes de informação utilizadas no cotidiano apresentaram relação de dependência $(\mathrm{p}=0,025)$, contudo, o mesmo não ocorreu quanto as fontes empregadas em momentos de quadros sintomáticos agudos não críticos ( $\mathrm{p}=0,119)$. Estes e demais achados são demonstrados na Tabela 2. 
Tabela 2. Associação e distribuição de variáveis relacionadas à comunicação em saúde quanto ao sexo biológico da população adscrita, Rio de Janeiro, RJ, Brasil, 2020.

\begin{tabular}{|c|c|c|c|c|c|}
\hline \multirow{3}{*}{ Variáveis } & \multicolumn{4}{|c|}{ Distribuição } & \multirow[t]{3}{*}{$\mathbf{p}$} \\
\hline & \multicolumn{2}{|c|}{$\mathbf{M}$} & \multicolumn{2}{|c|}{$\mathbf{F}$} & \\
\hline & $\mathbf{n}$ & $\%$ & $\mathbf{n}$ & $\%$ & \\
\hline Fontes de informações sobre saúde em momentos cotidianos & 47 & 100,0 & 153 & 100,0 & $0,02561^{\mathrm{a}}$ \\
\hline Profissionais de saúde & 29 & 61,7 & 90 & 58,8 & \\
\hline Google & 26 & 55,3 & 76 & 49,7 & \\
\hline Jornais impressos, online ou televisivos & 4 & 8,5 & 50 & 32,6 & \\
\hline Familiares, vizinhos e/ou amigos & 9 & 19,1 & 37 & 24,2 & \\
\hline Youtube & 8 & 17,0 & 31 & 20,3 & \\
\hline Facebook & 8 & 17,0 & 20 & 13,1 & \\
\hline WhatsApp & 5 & 10,6 & 18 & 11,8 & \\
\hline Instagram & 1 & 2,12 & 16 & 10,4 & \\
\hline Líder religioso & 7 & 14,9 & 15 & 9,8 & \\
\hline Twitter & 0 & 0 & 6 & 3,92 & \\
\hline Televisão & 4 & 8,5 & 1 & 0,65 & \\
\hline Ministério da Saúde & 0 & 0 & 1 & 0,65 & \\
\hline Fontes de informações sobre saúde em momentos sintomáticos agudos não críticos & 47 & 100,0 & 153 & 100,0 & $0,11970^{\mathrm{a}}$ \\
\hline Profissionais de saúde & 41 & 87,2 & 127 & 83,0 & \\
\hline Google & 20 & 42,5 & 45 & 29,4 & \\
\hline Familiares, vizinhos e/ou amigos & 14 & 29,8 & 37 & 24,2 & \\
\hline Líder religioso & 4 & 8,5 & 4 & 2,6 & \\
\hline Youtube & 2 & 4,2 & 12 & 7,8 & \\
\hline Não procuraria informação & 1 & 2,12 & 1 & 0,6 & \\
\hline Não soube responder & 0 & 0 & 4 & 2,6 & \\
\hline Percepção sobre confiança em fontes de informação em saúde & 47 & 100,0 & 153 & 100,0 & $0,1748^{b}$ \\
\hline Possui fonte de informação totalmente confiável & 23 & 48,9 & 57 & 37,3 & \\
\hline Não possui fonte de informação totalmente confiável & 24 & 51,1 & 96 & 62,7 & \\
\hline Fonte segura de informação & 23 & 100,0 & 57 & 100,0 & $0,04267^{\mathrm{a}}$ \\
\hline Internet & 2 & 8,69 & 6 & 10,5 & \\
\hline Instituições oficiais & 1 & 4,34 & 10 & 17,5 & \\
\hline Profissionais da Saúde & 4 & 17,4 & 22 & 38,6 & \\
\hline Unidade Básica de Saúde & 16 & 69,6 & 17 & 29,8 & \\
\hline Familiares e/ou vizinhos & 0 & 0 & 2 & 3,5 & \\
\hline
\end{tabular}

Legenda: a - Qui-quadrado; b - Teste exato de Fisher. Fonte: Dados do estudo. 
Desta forma, foi verificado que profissionais de saúde e o Google foram amplamente reportados como fontes de informação em saúde recorridas. Ademais, pessoas do sexo feminino reportaram o uso da mídia social Instagram® em maior frequência que os homens, enquanto os homens reportaram maior uso do Google $®$ tanto durante momentos cotidianos $(\mathrm{n}=26$; $55,3 \%)$, quanto em momentos sintomáticos leves ( $\mathrm{n}=20 ; 42,5 \%)$.

No que se refere a percepção sobre fontes de informações em saúde de confiança, foi demonstrado que pessoas do sexo masculino referem possuir fontes totalmente confiáveis com maior frequência do que pessoas do sexo feminino, contudo não houve significância estatística entre tais variáveis.

Foi possível identificar, ainda, que houve relação de dependência ( $\mathrm{p}=0,04)$, na qual o grupo do sexo feminino reportou maior confiança em fontes da Internet $(\mathrm{n}=06 ; 10,5 \%)$, meios de comunicação de instituições oficiais $(\mathrm{n}=10 ; 17,5 \%)$ e profissionais de saúde no geral, seja do âmbito privado ou do particular $(\mathrm{n}=22 ; 38,6 \%)$, contudo, houve considerável quantitativo que afirmou ter como fonte de ampla confiança a unidade básica de saúde ao qual é vinculado, principalmente entre os homens (sexo masculino 69,6\%; sexo feminino 29,8\%).

No quesito idade, as informações provenientes de meios de comunicação de instituições oficiais e da Internet no geral foram reportadas como de maior confiança por pessoas com média de idade de 29 anos $(\sigma=11,5)$, já informações provenientes de profissionais de saúde em geral (da rede privada ou particular) são vistas como de maior confiança por pessoas com média de idade de 40 anos $(\sigma=14,5)$. A unidade básica de saúde a qual os participantes são vinculados representou a fonte de informações mais confiável entre pessoas com média de idade de 43 anos $(\sigma=15,3)$. Tais dados são ilustrados na Tabela 3.

Diante de diversas estratégias de educação em saúde passíveis de serem implementadas e sua aceitação por parte dos usuários foi realizado delineamento de tal perfil, sendo este interpretado a partir da frequência com que os usuários afirmam acessar tais meios de comunicação, tais dados são demonstrados na Figura 3 e Tabela 3. Ademais, foram apresentadas seis afirmações aos participantes, relacionadas a crenças e convicções acerca de informações em saúde, para compreender o grau de concordância ou discordância e, consequentemente, a forma que a dinâmica com tais informações ocorrem na população estudada, sendo tais resultados apresentados na Tabela 3, contendo a distribuição dos dados por faixa etária, e Figura 4. 
Figura 3. Aceitação de diferentes meios de acesso à informação em saúde entre os usuários da unidade básica de saúde. Rio de Janeiro, RJ, Brasil, 2020.

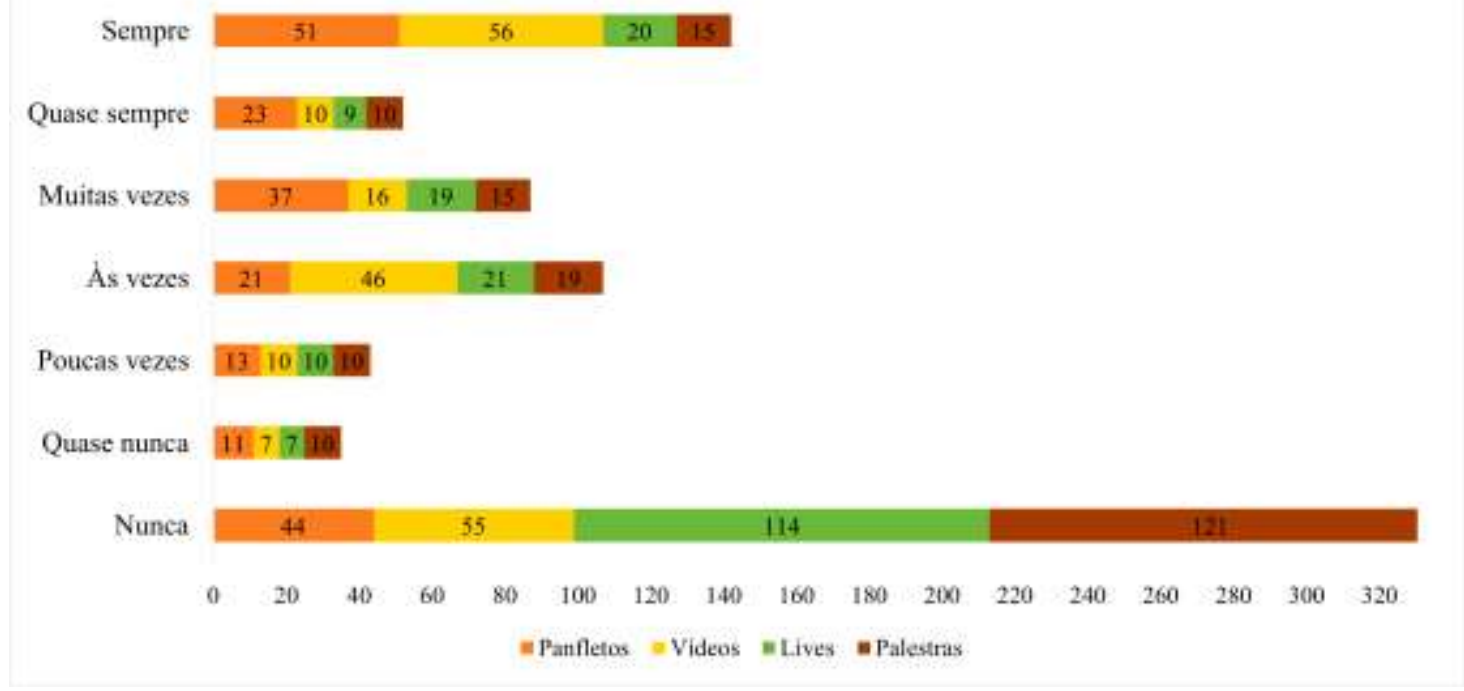

Fonte: Dados do estudo.

Conforme demonstrado na Figura 3, foi verificado que as palestras e as lives na Internet (vídeos transmitidos ao vivo) apresentaram menor aceitação e interesse, enquanto os panfletos informativos demonstraram discreta maior aceitação.

Ainda, a idade se mostrou como fator estatisticamente relevante em relação à aceitação de panfletos informativos sobre saúde $(\mathrm{p}=0,005)$, no qual os participantes com idade entre 18 a 35 anos demonstraram maior inclinação a aceitarem tal meio de comunicação, enquanto os indivíduos de 46 a 65 anos demonstraram menor inclinação.

Ademais, foi analisado o entendimento e as práticas dos usuários em relação à checagem de informações em seu cotidiano, sendo possível identificar que os usuários apresentam preocupação quanto a realização do referido procedimento. A frequência com que realizam as checagens se deu da seguinte forma: 26,5\% ( $\mathrm{n}=53)$ responderam que nunca realizam, $1 \%$ $(n=2)$ e $4 \%(n=8)$ responderam que realizam quase nunca e poucas vezes, respectivamente, $13 \%$ ( $n=26)$ realizam às vezes, $11 \%(n=26)$ e $13 \%(n=22)$ realizam muitas vezes e quase sempre, respectivamente, e 31,5\% $(n=63)$ sempre o fazem. No mais, houve correlação estatística entre a idade e a frequência de checagem das informações sobre saúde ( $p=0,015)$, de forma que os participantes de idade entre 18 a 25 anos demonstraram valorizar mais a checagem ("quase sempre" e "sempre") do que os participantes das demais faixas etárias, sendo tais distribuições demonstradas na Tabela 3.

Por último, foram apresentadas seis afirmações aos participantes, relacionadas a crenças e convicções acerca de informações em saúde, para compreender o grau de concordância ou discordância e consequentemente a forma que a dinâmica com tais informações ocorrem na população estudada. Os resultados são apresentados na Tabela 3 e Figura 4. 
Tabela 3. Associação e distribuição de variáveis relacionadas à comunicação em saúde quanto a faixa etária da população adscrita, Rio de Janeiro, RJ, Brasil, 2020.

\begin{tabular}{|c|c|c|c|c|c|c|c|c|c|c|}
\hline \multirow{3}{*}{ Variáveis } & \multicolumn{8}{|c|}{ Distribuição } & & \multirow{3}{*}{$\mathbf{p}$} \\
\hline & $\begin{array}{l}\text { Faixa } \\
\text { etária }\end{array}$ & $\mathbf{0}$ & 1 & 2 & 3 & 4 & 5 & 6 & $\begin{array}{c}\text { Amostra } \\
\text { total } \\
\text { n }(\%) \\
\end{array}$ & \\
\hline & \multicolumn{9}{|c|}{ n (\%) } & \\
\hline
\end{tabular}

graus de

concordância ${ }^{\dagger}$

Afirmação 1:
Somente
profissionais de
saúde são
qualificados
para realizar
orientações em
saúde
Afirmação 2:
Acredito em
todas as
orientações em
saúde que são
ditas a mim por
profissionais de
saúde
Afirmação $3:$
Acredito que a
experiência das
pessoas que já
vivenciaram
algum problema
de saúde é mais
confiável do
que a orientação
de um
profissional de
saúde

$\begin{array}{lllllllll}18-25 & 1(1,9) & 2(3,7) & 5(9,3) & 1(1,9) & 11(20,4) & 07(13,0) & 27(50,0) & 54(100,0) \\ 26-35 & 0(0,0) & 1(2,2) & 2(4,3) & 4(8,7) & 02(4,3) & 05(10,9) & 32(69,6) & 46(100,0) \\ 36-45 & 0(0,0) & 1(2,3) & 0(0,0) & 1(2,3) & 04(9,2) & 07(15,9) & 31(70,5) & 44(100,0) \\ 46-55 & 0(5,3) & 1(5,3) & 0(0,0) & 2(10,5) & 01(5,3) & 0(0,0) & 14(73,7) & 19(100,0) \\ 56-65 & 2(6,2) & 0(0,0) & 0(0,0) & 2(6,2) & 01(3,1) & 01(3,1) & 26(81,2) & 32(100,0) \\ 66-68 & 0(0,0) & 0(0,0) & 0(0,0) & 0(0,0) & 0(0,0) & 01(20,0) & 04(80,0) & 5(100,0)\end{array}$

$0,09784^{\mathrm{a}}$

$18-25 \quad 4(7,4) \quad 1(1,9) \quad 7(13,0) \quad 3(5,6) \quad 13(24,1) \quad 7(13,0) \quad 19(35,2) \quad 54(100,0)$

26-35 $2(4,3) \quad 0(0,0) \quad 3(6,5) \quad 3(6,5) \quad 11(23,9) \quad 10(21,7) \quad 17(37,0) \quad 46(100,0)$

36-45 $0(0,0) \quad 0(0,0) \quad 0(0,0) \quad 5(11,4) \quad 10(22,7) \quad 10(22,7) \quad 19(43,2) \quad 44(100,0)$

$\begin{array}{lllllllll}46-55 & 2(10,5) & 0(0,0) & 0(0,0) & 3(15,8) & 1(5,3) & 2(10,5) & 11(57,9) & 19(100,0)\end{array}$

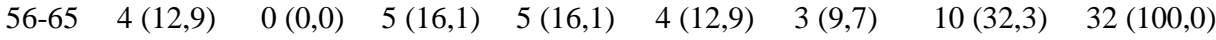

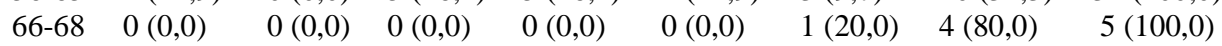

$0,19300^{\mathrm{a}}$

$\begin{array}{lllllllll}18-25 & 19(35,2) & 6(11,1) & 3(5,6) & 4(7,4) & 6(11,1) & 4(7,4) & 12(22,2) & 54(100,0) \\ 26-35 & 18(39,1) & 1(2,2) & 2(4,3) & 1(2,2) & 8(17,4) & 5(10,9) & 11(23,9) & 46(100,0) \\ 36-45 & 15(34,1) & 1(2,3) & 4(9,1) & 8(18,2) & 2(4,5) & 6(13,6) & 8(18,2) & 44(100,0) \\ 46-55 & 9(47,4) & 1(5,3) & 0(0,0) & 2(10,5) & 2(10,5) & 2(10,5) & 3(15,8) & 19(100,0) \\ 56-65 & 6(18,8) & 1(3,1) & 0(0,0) & 2(6,2) & 3(9,4) & 3(9,4) & 17(53,1) & 32(100,0) \\ 66-68 & 0(0,0) & 1(20,0) & 0(0,0) & 2(40,0) & 1(20,0) & 0(0,0) & 1(20,0) & 5(100,0)\end{array}$

$0,03568^{\mathrm{a}}$

Afirmação 4:

Entendo tudo

que os

profissionais de

saúde me falam

Afirmação 5:

Não tenho

vergonha de

pedir aos

profissionais de

saúde que

esclareçam

dúvidas ou

repitam

informações

Afirmação 6:

É muito

importante

checar as fontes

das informações
$18-25 \quad 1(1,9)$

26-35 $1(2,2)$

$36-45 \quad 0(0,0)$

46-55 $\quad 0(0,0)$

56-65 $0(0,0)$
$1(1,9) \quad 5(9,3)$

$0(0,0) \quad 2(4,3)$

$0(0,0) \quad 0(0,0)$

$1(5,3) \quad 0(0,0)$

$1(3,1) \quad 4(12,5) \quad 2(6,2)$
$6(11,1) \quad 2(3,7)$

$4(7,4)$

$3(6,5)$

$2(4,5)$

(88,6) $\quad 44(100,0)$

$\begin{array}{llll}(5,3) & 2(10,5) & 14(73,7) & 19(100,0)\end{array}$

$1(3,1) \quad 3(9,4) \quad 21(65,6) \quad 32(100,0)$

$0,00004091^{\mathrm{a}}$ 


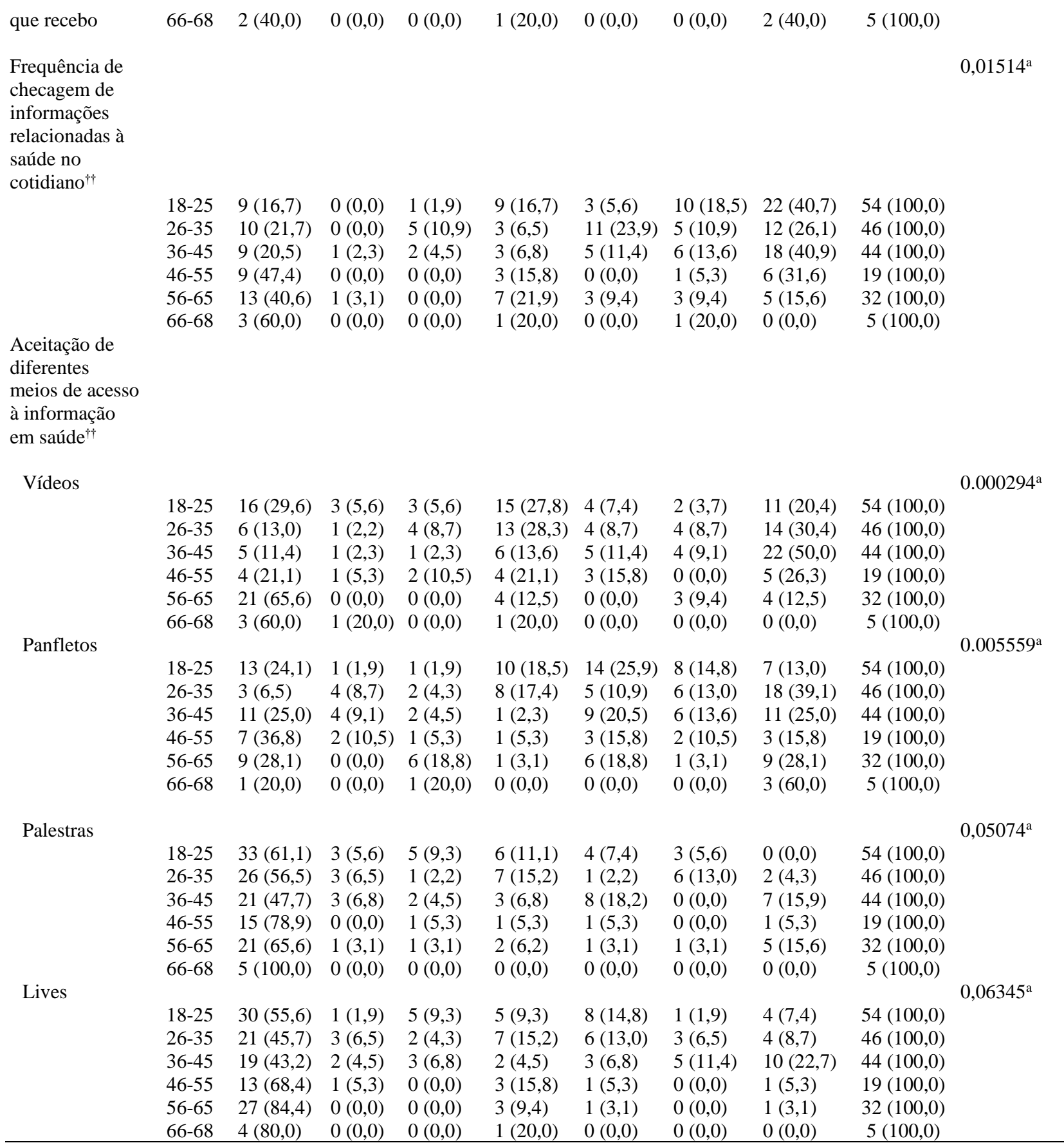

Legenda: a - Teste de Qui-quadrado. ${ }^{\dagger}$ Categorias de graus de concordância: (0) Discordo totalmente; (1) Discordo muito; (2) Discordo em parte; (3) Não concordo nem discordo (4) Concordo em parte; (5) Concordo muito; (6) Concordo totalmente. ${ }^{++}$Categorias do quesito frequência: (0) Nunca; (1) Quase nunca; (2) Poucas vezes; (3) Às vezes; (4) Muitas vezes (5) Quase sempre; (6) Sempre. Fonte: Dados do estudo.

A afirmação 3 foi impactada pelo fator idade ( $\mathrm{p}=0,03568)$, de forma que os indivíduos de 18 a 45 anos apresentaram maior discordância do que a faixa etária de 46-68 anos. As afirmações 4 e 6 também apresentaram distribuições diferentes relacionadas à faixa etária dos participantes. No que se relaciona ao entendimento dos usuários quanto ao que é comunicado pelos profissionais de saúde (afirmação 4), as faixas etárias de 36 a 55 anos afirmaram ter amplo entendimento do que é dito, obtendo destaque dentre as demais faixas etárias ( $\mathrm{p}=0,00004091)$. As demais afirmativas não demonstraram ser impactadas pelo fator idade. 
Figura 4. Grau de concordância/discordância quanto às afirmações sobre o processo de comunicação em saúde. Rio de Janeiro, RJ, Brasil, 2020.

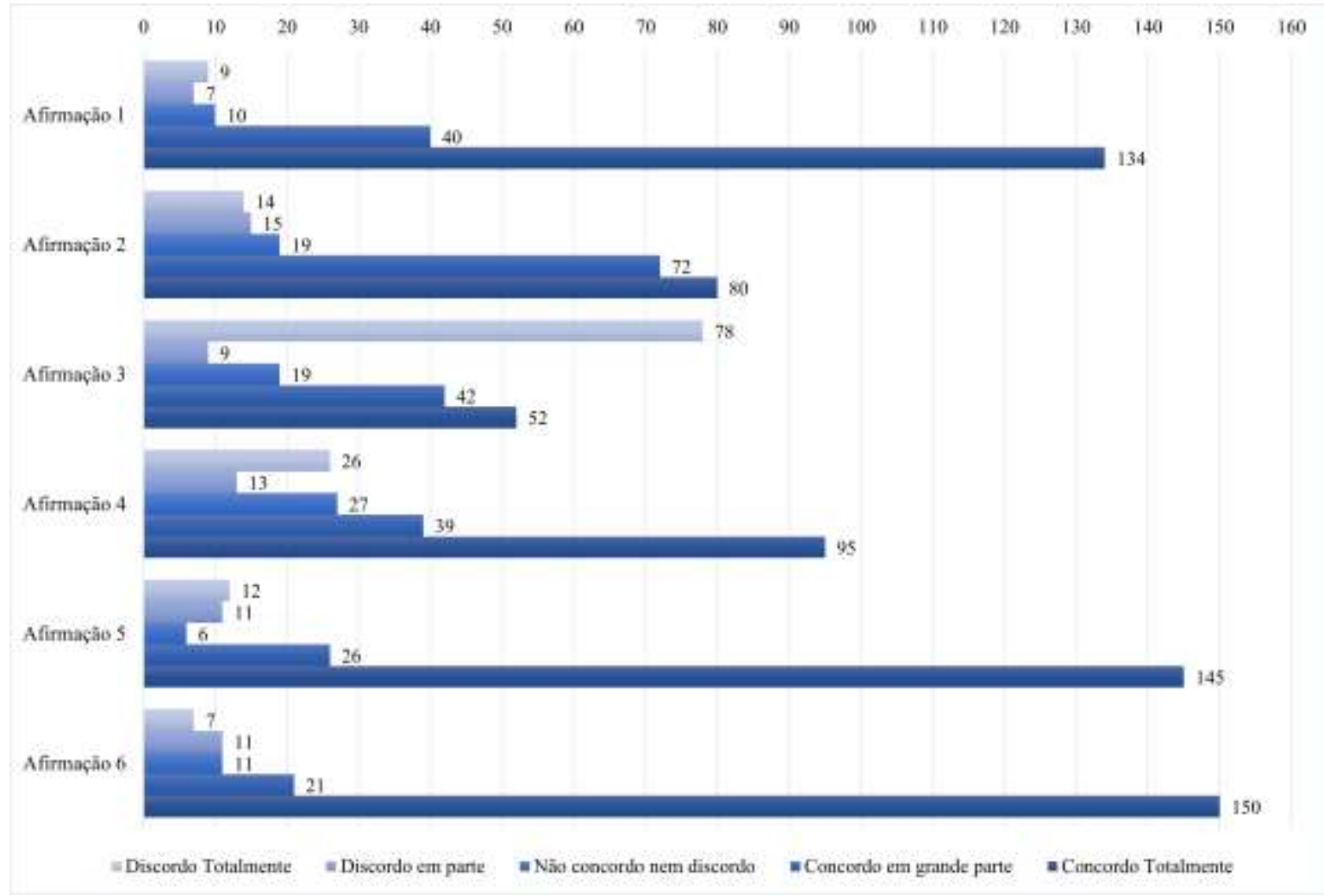

Legenda: Afirmação 1: Somente profissionais de saúde são qualificados para realizar orientações em saúde; Afirmação 2: Acredito em todas as orientações em saúde que são ditas a mim por profissionais de saúde; Afirmação 3: Acredito que a experiência das pessoas que já vivenciaram algum problema de saúde é mais confiável do que a orientação de um profissional de saúde; Afirmação 4: Entendo tudo que os profissionais de saúde me falam; Afirmação 5: Não tenho vergonha de pedir aos profissionais de saúde que esclareçam dúvidas ou repitam informações; Afirmação 6: É muito importante checar as fontes das informações que recebo. *Observação: originalmente os dados continham seis categorias, conforme descrito na Tabela 3, sendo os dados das categorias 0-1 e 5-6 agrupados para melhor visualização do leitor. Fonte: Dados do estudo.

\section{Discussão}

Para o desenvolvimento de estratégias eficazes de comunicação em saúde é necessário avaliar e compreender o público-alvo ao qual se pretende alcançar a partir de fatores individuais e coletivos. Desta forma, a presente discussão se estrutura a partir das perspectivas sobre perfil sociodemográfico da população adscrita, devido ao seu impacto como fatores condicionantes e determinantes, perfil de acesso às tecnologias da informação e comunicação e, por sua vez, de fatores específicos do processo de comunicação em saúde desta população.

\section{Perfil sociodemográfico e de acesso às Tecnologias da Informação e Comunicação}

Os resultados mostraram que pessoas do sexo feminino são predominantes no que se refere à utilização da APS, assim como as faixas etárias de 18-29 anos e 40-59, vindo de encontro ao estudo de Perillo (Perillo et al., 2020).

A literatura aponta que condições de baixa renda e escolaridade são contribuintes para o menor acesso à saúde, contudo, no presente estudo pessoas sob tais condições representaram a maioria das participantes. Desta forma, é reforçado o entendimento de que a Estratégia de Saúde da Família desempenha papel primordial para promoção do acesso à saúde às populações vulneráveis e para diminuição de iniquidades sociais (Malta et al., 2017; Poças, Freitas, \& Duarte, 2017; Stopa et al., 2017). 
Estudos demonstram que a procura pelos serviços de saúde está diretamente relacionada aos problemas de saúde apresentados pelo indivíduo, e que pacientes com doenças crônicas não transmissíveis são os usuários mais presentes nos serviços de saúde (Malta et al., 2017; Stopa et al., 2017). Desta forma, justifica-se o predomínio da procura por atendimento relacionado ao tratamento para Hipertensão Arterial e Diabetes Mellitus no presente estudo.

Neste estudo, usuários com as menores rendas foram os que apresentam menor posse de aparelhos eletrônicos, sendo a combinação de televisão e celular a mais reportada. Ainda, o smartphone foi o aparelho de Tecnologia da Informação e Comunicação (TIC) mais reportado pelos participantes. Tal dado é corroborado com pesquisa que indica o smartphone como a TIC mais utilizada no Brasil em 2020, sendo a proporção de um aparelho para cada habitante (Meirelles, 2020).

Em 2018, o Instituto Brasileiro de Geografia e Estatística (IBGE) demonstrou que 99,2\% dos domicílios estudados possuíam acesso à Internet, 93,2\% possuíam aparelho celular e 96,4\% aparelho de televisão. No entanto, a minoria dos domicílios possuía microcomputadores $(41,7 \%)$ e tablets (12,5\%). De forma semelhante, no presente estudo, a maioria dos usuários da UBS possuíam acesso à Internet, aparelho celular e aparelho de televisão, enquanto a minoria possuía computadores, notebooks e tablets (IBGE, 2018).

A partir de tais dados é possível criar estratégias empregando as TICs conforme o acesso do público-alvo. Assim, meios de se estabelecer comunicação com a população que sejam possíveis através de aparelhos celular, tornam-se ferramentas preciosas para o processo de educação em saúde. Em contrapartida, criar estratégias que necessitem de dispositivos mais robustos, pode se tornar um empecilho para o sucesso do processo. De igual forma, considerar fatores como escolaridade e sexo biológico se torna imprescindível para adequação da linguagem e estratégias de ensino-aprendizagem.

\section{Processo de Comunicação em Saúde}

A compreensão acerca do processo de comunicação em saúde vem a ser uma ferramenta valiosa para implementação de estratégias para seu aprimoramento. Desta forma, a elucidação sobre os diversos aspectos envolvidos nesta dinâmica, conforme o contexto o qual se está inserido, torna-se agente facilitador para a identificação de meios para alcance de um otimizado e eficiente processo comunicativo.

Deste ponto de vista, identificar os meios de comunicação de maior aceitação do público-alvo pode contribuir para o aumento da adesão e evitar a utilização de recursos de forma ineficiente. A partir desta perspectiva, foi possível constatar a utilização de panfletos e vídeos como os meios mais aceitos entre a população adscrita. Por sua vez, tais estratégias são amplamente apontadas na literatura como experiências exitosas para a educação em saúde em diversos temas, tais como, promoção de hábitos saudáveis e do conhecimento acerca de doenças relevantes no contexto das unidades básicas de saúde, como a diabetes mellitus e a febre amarela (Marchetti \& da Silva, 2020; Silva et al., 2020; Silva \& Terencio, 2019; Souza et al., 2019).

No que se refere ao perfil de utilização da Internet para aquisição de informações sobre saúde, semelhantemente ao presente estudo, uma pesquisa conduzida com 1.828 pessoas, encontrou o dado de que a maioria de seus participantes (90\%) a empregavam para compreender problemas de saúde próprios (90\%) e de seus familiares (79\%). Ademais, busca por temas sobre alimentação saudável, medicamentos e condições específicas de saúde foram identificadas como de grande interesse dos participantes, vindo de encontro aos achados do presente estudo (Moretti, Oliveira \& da Silva, 2012). Desta forma, a partir de tal informação é possível delinear estratégias de educação em saúde direcionadas aos temas mais relevantes e de maior interesse entre a população adscrita.

Contudo, na referida pesquisa, a Internet obteve a maior frequência em relação as principais fontes de informação sobre saúde empregadas entre os participantes (86\%), sendo os profissionais de saúde fontes de informações menos valorizadas (74\%). No presente estudo, os profissionais de saúde foram os mais reportados como fonte de informações, tanto no contexto 
cotidiano $(\mathrm{n}=119 ; 59,5 \%)$ como no contexto de quadros agudos $(\mathrm{n}=168 ; 84,5 \%)$, entretanto o uso do Google, Youtube e WhatsApp também foram reportados. O estudo ainda traz a mudança ocorrida na dinâmica entre paciente e profissional de saúde devido ao acesso aumentado às informações em saúde, no qual o profissional não é mais detentor exclusivo do conhecimento e que, neste novo cenário, são necessárias negociações e debates em um atendimento em saúde (Moretti, Oliveira \& da Silva, 2012).

No ano de 2018, o Brasil ocupava a segunda posição entre os países que mais possuíam usuários do Instagram, sendo as mulheres a maioria entre os usuários ativos da referida rede social (59\%) (We Are Social, 2018). De forma similar, no presente estudo, as mulheres reportaram o uso do Instagram como meio mais utilizado para pesquisas relacionadas à saúde, demonstrando o potencial de utilização das redes sociais para estratégias de educação em saúde.

No cenário de comunicação em saúde, um tema de grande relevância consiste na geração de Fake News (Monari \& Bertolli Filho, 2019). Neste sentindo, compreender a percepção da população adscrita sobre fatores que permeiam este tema auxiliam na determinação de estratégias de educação em saúde mais realistas e específicas para o público-alvo.

No presente estudo foi verificado que os participantes mais jovens demonstraram maior confiança em informações provenientes da Internet, no entanto, conforme o aumento da faixa etária as fontes tradicionais eram mais valorizadas, tais como profissionais de saúde da rede pública e privada. De forma semelhante, em estudo conduzido com 169 jovens sobre fake news, a maioria dos participantes demonstrou confiar em notícias oriundas da Internet (páginas da Internet, Wikipédia e canais da plataforma Youtube®) (Jacobi, 2019).

Apesar de tais dados, quando questionados quanto a credibilidade dos profissionais de saúde, foi possível identificar que a maioria dos participantes veem os profissionais de saúde como única fonte qualificada para realizar orientações em saúde ( $87 \% ; n=174)$ e acreditam fortemente nas informações por eles disponibilizadas ( $76 \% ; n=152)$, sendo tal dado corroborado por estudo em que os profissionais de saúde obtiveram maior índice de confiança dentre os entrevistados (76\%) em relação às demais fontes de informação em saúde apresentadas (Moretti, Oliveira \& da Silva, 2012).

Ainda, os participantes do presente estudo demonstraram compreender a importância de checar as fontes das informações recebidas, no qual 85\% (n=171), afirmou ser muito importante tal procedimento, contudo não há garantias de que o procedimento é de fato realizado no cotidiano e se é realizado da forma adequada, enfatizando a demanda pelo papel educador da APS neste contexto. Estudo realizado sobre fake news demonstrou que 58,17\% de seus participantes ( $\mathrm{n}=169)$ sentiam insegurança quanto a verificação da veracidade de determinada informação e que aproximadamente 64,5\% poderiam já ter compartilhado notícias falsas sem intenção, devido ao não conhecimento da natureza da informação (Jacobi, 2019).

A afirmação 3 (acredito que a experiência das pessoas que já vivenciaram algum problema de saúde é mais confiável do que a orientação de um profissional de saúde) diz muito sobre a vulnerabilidade em relação às fake news, visto que estudos sobre o tema apontam que sua base se dá a partir da maior valorização de crenças pessoais em detrimento da lógica e dos fatos, a chamada autoridade experiencial (Llorente, 2017; Monari \& Bertolli Filho, 2019; Sacramento, 2018).

Desta forma, apesar de grande quantitativo de participantes reafirmarem a credibilidade de profissionais de saúde e a importância de checar informações, por sua vez uma quantidade significativa (47\%; n=94) demonstrou valorizar experiências pessoais em detrimento de informações fornecidas por profissionais da área, enquanto menor quantitativo (39\%; n=78) não se apoia nessas experiências primordialmente. Neste grupo, pessoas de maior faixa etária (46-68 anos) demonstraram se apoiar mais em experiências pessoais em detrimento das informações fornecidas por profissionais de saúde, sendo, portanto, um grupo que requer maior atenção neste quesito nas propostas de educação em saúde.

No mais, além da fonte da informação, sua confiabilidade e acesso, há de se atentar a qualidade com que a mensagem é comunicada, visto que barreiras podem se interpor no processo comunicativo o prejudicando (Borba, Santos, \& Puggina, 2017). Portanto, é de grande importância o entendimento da dinâmica de comunicação entre profissional de saúde e paciente. 
Assim, o presente estudo demonstrou que a maioria dos usuários da UBS possuem um bom entendimento quanto as informações disponibilizadas pelos profissionais de saúde $(67 \%$; $=134)$, contudo, um quantitativo considerável demonstrou não alcançar o mesmo nível de compreensão (19,5\%; n=39), sendo um fator preocupante para o processo de comunicação.

Entretanto, maior quantitativo de usuários afirmou não sentir constrangimento ao solicitar retirada de dúvidas ou repetição de informações por parte dos profissionais de saúde $(85,5 \%$; $=171)$, sendo tal atitude fundamental e facilitadora para o adequado processo de comunicação em saúde.

Tais questões sobre a comunicação são comuns na prática clínica. Em estudo sobre avaliação da comunicação médico-paciente, mensurada através de pontuações atribuídas pelos pacientes às consultas ambulatoriais com profissionais médicos, foi verificado que os menores scores foram relacionados a aspectos de compreensão acerca de informações, sendo os principais fatores problemáticos apontados na pesquisa: explicação sobre medicamentos, explicação acerca de exames, incentivo à realização de perguntas e respostas às perguntas e preocupações do paciente (Braga \& Carozzo, 2020).

\section{Conclusão}

O presente estudo apresentou subsídios para o desenvolvimento de estratégias de comunicação em saúde direcionadas a uma população adscrita de uma clínica da família do município do Rio de Janeiro, a partir da identificação de aspectos sociodemográficos e da realização de um diagnóstico situacional sobre o processo de comunicação em saúde.

Foi constatado que a idade se constitui como fator relevante no que se refere a estratégia a ser empregada para comunicação em saúde, visto que a confiança depositada sobre as informações sobre saúde recebidas, assim como o meio de divulgação, são influenciadas por tal fator. Além disso, foi evidenciada a potencialidade de atuação da APS em relação à orientação quanto ao consumo de informações sobre saúde, no sentido de fornecer instruções para minimizar a circulação de fake news e os prejuízos potenciais para a saúde de sua população adscrita.

Este estudo contribui para o planejamento de ações de educação em saúde por profissionais assistenciais da Atenção Primária à Saúde e opera enquanto agente motivador para que demais diagnósticos situacionais sejam realizados pelas unidades básicas de saúde com base em suas populações adscritas.

Contudo, o estudo possui como limitações população amostral reduzida e vieses relacionados ao contexto de pandemia por COVID-19, inviabilizando maior variedade de respostas oriundas de diferentes grupos usuários da unidade de saúde. Desta forma, recomenda-se a realização de novos estudos com maior número amostral e em diferentes contextos epidemiológicos.

\section{Referências}

Borba, A., Santos, B. M., \& Puggina, A. C. G. (2017). Barreiras de comunicação nas relações enfermeiro-paciente: revisão integrativa. Revista Saúde - UNGSer, 11(1/2), 48-61.

Braga, R. L., \& Carozzo, N. P. P. (2020). Avaliação da comunicação médico-paciente na perspectiva de ambos interlocutores. Salud (i) Ciencia, 23(i), 668672 .

Brasil. Ministério da Saúde. Secretaria de Atenção à Saúde. (2013). Portaria no 2.761, de 19 de novembro de 2013. Institui a Política Nacional de Educação Popular em Saúde no âmbito do Sistema Único de Saúde (PNEPS-SUS). Diário Oficial da União. Brasília, DF.

Brasil. Ministério da Saúde. Secretaria de Atenção à Saúde. (2017). Portaria no 2.436, de 21 de setembro de 2017. Aprova a Política Nacional de Atenção Básica, estabelecendo a revisão de diretrizes para a organização da Atenção Básica, no âmbito do Sistema Único de Saúde (SUS). Diário Oficial da União. Brasília, DF.

Coscrato, G., \& Bueno, S. M. V. (2013). Concepts of health education by public health nurses. Revista da Escola de Enfermagem da USP, 47(3), 714-721.

IBGE. Instituto Brasileiro de Geografia e Estatística. (2018). Acesso à internet e à televisão e posse de telefone móvel celular para uso pessoal 2018. Recuperado 22 de dezembro de 2020, de https://biblioteca.ibge.gov.br/index.php/biblioteca-catalogo?view=detalhes\&id=2101705

Jacobi, G. (2019). Mídias sociais como fonte de informação de adolescentes e jovens em tempos de fake news. [Trabalho de Conclusão de Curso] Porto Alegre: Universidade Federal do Estado do Rio Grande do Sul. 
Llorente J. A. (2017). A era da pós-verdade: realidade versus percepção. Revista UNO, 27. Recuperado 22 de dezembro de 2020, de https://www.revistauno.com.br/numero-27/

Malta D. C., Bernal, R. T. I., Lima, M. G., de Araújo, S. S. C., da Silva, M. M. A., Freitas, M. I. de F., \& Barros, M. B. de A. (2017). Noncommunicable diseases and the use of health services: Analysis of the National Health Survey in Brazil. Revista de Saúde Publica, 51, 1S-10S.

Marchetti, J. R., \& da Silva, M. (2020). Educação em saúde na atenção primária: Diabetes Mellitus. Anuário Pesquisa E Extensão Unoesc Xanxerê, 5 , e24183.

Medronho, R. A., Bloch K. V., Luiz R. R., \& Werneck G. L. (2008). Epidemiologia. Atheneu

Meirelles, F. S. (2020). Pesquisa Anual do Uso de TI. FGV, 162. https://eaesp.fgv.br/producao-intelectual/pesquisa-anual-uso-ti

Ministério da Saúde. (2015). Documento Orientador de apoio aos debates da 15ª Conferência Nacional de Saúde. Conselho Nacional de Saúde. Brasília: DF: Ministério da Saúde

Miranda, F. S., \& Rocha, D. G. (2018). O uso do Facebook na promoção da saúde: uma revisão bibliográfica sobre empoderamento e participação popular. Revista Eletrônica de Comunicação, Informação e Inovação em Saúde, 12(2), 232-243.

Monari, A. C. P., \& Bertolli Filho, C. (2019). Saúde sem Fake News: estudo e caracterização das informações falsas divulgadas no Canal de Informação e Checagem de Fake News do Ministério da Saúde. Revista Mídia e Cotidiano, 13(1), 160.

Moretti, F.A., Oliveira V.E., \& da Silva, E. M. K. (2012). Acesso a informações de saúde na internet: uma questão de saúde pública? Revista da Associação Médica Brasileira, 58(6), 650-658.

Morettin, P. A. \& Bussab, W. O. (2010). Estatística Básica. Saraiva.

Neto, M., Gomes, T. de O., Porto, F. R., Russo Rafael, R. de M., Fonseca, M. H. S., \& Nascimento, J. (2020). Fake news no cenário da pandemia de Covid-19. Cogitare Enfermagem, 25(0).

Perillo, R. D., Poças, K. C., Machado, I. E., Bernal, R. T. I., Duarte, E. C., \& Malta, D. C. (2020). Fatores associados à utilização da atenção primária pela população adulta de Belo Horizonte, Minas Gerais, segundo inquérito telefônico. Revista Mineira de Enfermagem, 24(0), 1-10.

Poças, K. C., Freitas, L. R. S. de, \& Duarte, E. C. (2017). Censo de estrutura da Atenção Primária à Saúde no Brasil (2012): estimativas de coberturas potenciais. Epidemiologia e serviços de saúde, 26(2), 275-284.

Sacramento, I. (2018). A saúde numa sociedade de verdades. Revista Eletrônica de Comunicação, Informação e Inovação em Saúde, 12(1).

Shu, K., Sliva, A., Wang, S., Tang, J., \& Liu, H. (2017, agosto 6). Fake news detection on social media: A data mining perspective. arXiv, 19, 22-36.

Silva, C. V., Pinheiro, F. A., Batista T.L., Guerra N. A. Q., Souza T. V. T. S, Gomes R. K. G. (2019). Educação em saúde na atenção primária : aplicação de um panfleto informativo sobre febre amarela. Mostra Interdisciplinar Do Curso De Enfermagem, 3(1).

Silva, E. A. da, \& Terencio, M. L. (2019). Educação em Saúde: a utilização de panfletos informativos e educativos como estratégia de educação e prevenção às patologias mais prevalentes da população no contexto da atenção básica [Artigo completo]. In Universidade Federal da Integração Latino-Americana Artigos Científicos, II Semana Integrada de Ensino, Pesquisa e Extensão (p.217-221).

Silva, E. de S. M. e, Ono, B. H. V. S., Souza, J. C., \& Menin, I. B. F. (2020). Media and health promotion in times of COVID-19. Research, Society and Development, 9(8), e842986252.

Silva, S. C., Ferro, E. L. B. S., Pereira, C. H. C., Ramos, S. B., \& Manochio-Pina, M. G. (2020). Vídeos educativos de oficina culinária para incentivo da alimentação saudável em Unidades de Saúde. Brazilian Journal of Development, 6(4), 21316-21336.

Souza, M. do S. de, \& Cabral, I. E. (2014). 25 years of Unified Health System Regulamentation and the 15 th National Conference of Health. Escola Anna Nery, 18(3), 376-378.

Starfield, B., Shi, L., \& Macinko, J. (2005). Contribution of primary care to health systems and health. Milbank Quarterly, 83, 457-502.

Stopa, S. R., Malta, D. C., Monteiro, C. N., Szwarcwald, C. L., Goldbaum, M., \& Cesar, C. L. G. (2017). Use of and access to health services in Brazil. Revista de Saúde Publica, 51, 1S-10S

Tasca, R., Massuda, A., Carvalho, W. M., Buchweitz, C., \& Harzheim, E. (2020). Recommendations to strengthen primary health care in Brazil. Revista Panamericana de Salud Publica, 44.

We Are Social. (2018). Digital in 2018 in Southern America Part 1 - North. https://www.slideshare.net/wearesocial/digital-in-2018-in-southern-america-part1-north-86863727 